\title{
Optimal positioning of irregular shapes in stamping die strip
}

\author{
Roberto Licari • E. Lo Valvo
}

Received: 25 November 2009 / Accepted: 2 June 2010 /Published online: 20 June 2010

(C) Springer-Verlag London Limited 2010

\begin{abstract}
The nesting of two-dimensional shapes is a common problem, where raw material has to be economically cut. As for the single-pass single-row strip layout, several algorithms, based on established methods, have been proposed. Moreover, it should be noticed that the optimum layout should also consider a few constraints, like grain orientation for subsequent forming operation, correct bridge width, and the commercial roll of metal width in order to make solutions applicable in real industrial environments. Most of the procedures until now shown in literature are quite complex and often ignore these real constraints. They usually make use of sliding techniques and are not able to effectively work with relatively multiple-connected figures. In particular, most of the different proposed procedures are based on the No Fit Polygon (NFP) computation of non-convex polygons, which often generates holes. This work is a proposal for a more efficient method, which can be used in heuristic procedures. In order to overcome some faults of most of the former methods presented in literature, in this paper a new geometric entity called "No Fit Path" (NFPh) of nonconvex polygons is applied. It allows researchers to find solutions of nesting problems even when there are NFP faults due to degenerate solutions. Moreover, the No Fit Path allows researchers to easily read, modify, or share their results, overcoming all those problems arising from the usual large amount of information and from the different origins and formats of the obtained data. Given two nonconvex polygons, the algorithm is able to calculate their
\end{abstract}

R. Licari $(\bowtie) \cdot$ E. Lo Valvo

Dipartimento di Tecnologia Meccanica,

Produzione ed Ingegneria Gestionale, University of Palermo,

viale delle Scienze,

Palermo 90128, Italy

e-mail: rlicari@dtpm.unipa.it
NFPh very quickly and without any approximation by a polygon clipping method. In this paper a totally automated procedure has been developed. This procedure firstly obtains the "No Fit Path" (NFPh); secondly, between all the existing positions on the NFPh, the algorithm searches the optimal one, minimizing the global waste. The proposed approach also allows designers to set an optimal orientation of the shapes on the roll of metal, taking account of the grain orientation in order to obtain the best mechanical characteristics for the cut pieces.

Keywords Nesting · Single-pass single-row layout · Stamping die strip $\cdot$ Part layout $\cdot$ NFP

\section{Introduction}

How to perform cutting and packing problem of irregular shapes is a fundamental problem, presenting different solutions and applications in the wide variety of industrial sectors.

As a matter of fact, it occurs not only in typical mechanical industries, but also in leather, wood and textile factories, in goods' storage cases, or in the layout design of newspapers or magazines. This is why a great number of industries are always more frequently facing the problem of nesting which is how to economically cut two-dimensional irregular shapes from a given stock sheet. An irregular polygon can have sides of any length and each interior angle can be any measure. They can be convex or concave, but all concave polygons are irregular since the interior angles cannot all be the same.

A common problem as far as stock cutting is concerned is how to obtain the best nesting of some pieces of flat patterns which occupy a minimal area, in accordance with a 
number of liabilities. As known, among all the problems encountered, the main target is the optimal positioning of the shapes on the commercial roll of metal in order to cut them as economically as possible (Fig. 1).

Moreover, in the die layout design for sheet metal parts, a large number of identical shapes are produced by cutting a metal strip. Since a great quantity of items is usually produced, even a very small inefficiency in material utilization causes a large amount of material waste. The best material utilization is based on the right orientation of the shapes on the strip: the best layout is the one obtaining the highest level of material utilization.

There are other important goals to be achieved. One is to save waste materials - especially when they are rare or very expensive. Another is the possibility of obtaining a suitable solution even in a case of complex shapes with holes or an exact fit; finally to respect the shapes' correct disposition on the strip when the grain orientation is significant.

Traditionally, nesting layouts were carried out manually but this kind of approach is a very hard and timeconsuming process. Depending on the designer's skills and experience and the complexity of the shapes, an acceptable layout is not always obtained and the optimal one is almost impossible to obtain. For this reason, as computers become common, powerful, and cheap, in recent years, computer-aided software tools have been implemented and used to carry out the nesting of part blanks automatically. Due to the increasing importance of cutting and packing problems, in the last years several categories of them have been described. They provided an excellent instrument for the organization and categorization of existing and new literature. However, over the years also some deficiencies of these typologies became evident, which created problems in dealing with recent developments and prevented it from being accepted more generally. Wäscher et al. [1] proposed an improved typology, which is partially based on precedent ideas, but they introduced new categorization criteria, which define problem categories different from those in literature.

In the last year, the problem of cutting and packing irregular shapes has become so important and wide that Bennel et al. [2] has set out to provide a tutorial covering the core geometric methodologies currently employed by

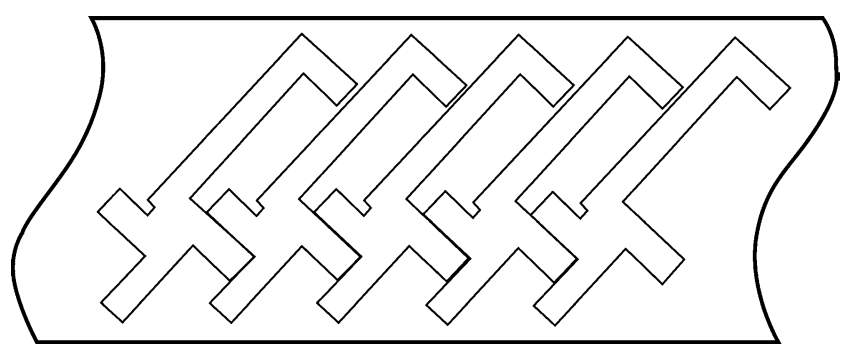

Fig. 1 Disposition of items on a strip researchers in the cutting and packing of irregular shapes. In the past some authors have faced this problem by using a tool called No Fit Polygon (NFP) [3-7].

In a recent work by Lam et al. [7], the evaluation of the well known Minkowski sum has been used, in order to achieve an efficient nesting of part blanks. In the part layout formation, authors calculated the strip pitch and width for different blank-pair orientations. Their target was to obtain the optimum orientation of two nested pairs that results in the greatest material utilization. These algorithms for nesting and part layout formation were implemented in a CAD environment and some case studies were carried out on typical parts to demonstrate the method discussed. However, the method sometimes faulted or carried out some non-optimal solutions.

Unfortunately, when the problem concerns complex shapes, NFP is often not able to reach all the desired targets; for this reason, in this paper the No Fit Path (NFPh) concept is used and analyzed instead of NFP in order to overcome this restriction. Sometimes a shape-even a simple shape cut from a regular stock - has to be folded later: in this case the goal is also to respect the grain orientation of the stock [8].

\section{Problem review}

In the stamping operation, production costs are dominated by material costs, so even tiny per part gains in material utilization are worth pursuing. In stamping, sheet metal parts of various levels of complexity are produced rapidly, often in very high volumes, using hard tooling. The production process operates efficiently, and material costs can typically represent $75 \%$ of total operating costs in a stamping facility [9]. Not all of this material is used in the parts, however, due to the need to trim scrap material from around irregularly shaped parts. The amount of scrap produced is directly related to the efficiency of the stamping strip layout. Clearly, using optimal strip layouts is crucial to a stamping firm's competitiveness.

The degree of this trim loss is determined at the tooling design stage when the strip layout is created. As a part or parts are laid out on the strip, the designer chooses the orientation of the part(s), width of the strip, and, in the case of multiple parts blanked together, their relative positions. Ideally, the material utilization is maximized. The value of even tiny improvements in material utilization can be great; for example, in a stamping operation running at 200 strokes per minute, a savings of just $10 \mathrm{~g}$ of material per part will accumulate into a savings of more than a ton of raw material per 8-h shift. The material utilization is set during the tooling design stage and remains fixed for the (usually 
long) life of the tool. Thus, there is significant value in determining the optimal strip layout before tooling is built.

This task is complicated, however, since changing each variable in the layout can change both the pitch (distance along the strip between adjacent parts) and strip width simultaneously. Evaluating layout efficiency manually is extremely challenging, and while exact optimal algorithms have been described for the layout of a single part on a strip, so far only approximate algorithms have been available for the layout of pairs of parts together. Nesting solutions for pairs of parts is an important problem since it is empirically known that nesting pairs of parts can often improve material utilization compared to nesting each part on a separate strip. This paper addresses both the common cases in which a given part is nested with a second copy of itself and when two or more different parts are nested together.

The No Fit Polygon (NFP) has become an increasingly popular option for dealing with the geometry since it is more efficient than direct trigonometry, particularly when using an iterative search, yet sharing the benefit of accuracy by using the original edges of the polygon (Fig. 2) [10-13].

The concept opens up new options for placement strategies. In essence, the NFP is a polygon derived from the relationship between two polygons. As a matter of fact, given two polygons $\mathrm{A}$ and $\mathrm{B}$ such as the position of $\mathrm{A}$ and the orientation of $\mathrm{B}$ are all fixed, then the NFP of B relative to A completely describes all those positions where a reference point (RP) of B polygon (say orbiting polygon) can be placed in order to have B touching A polygon (say stationary polygon) without overlapping. Efficient methods to calculate the NFP of two convex polygons or one convex and one non-convex polygon have been developed by other researchers. However, when both polygons are non-convex, the current methods of calculation are inefficient or noneasy to implement.

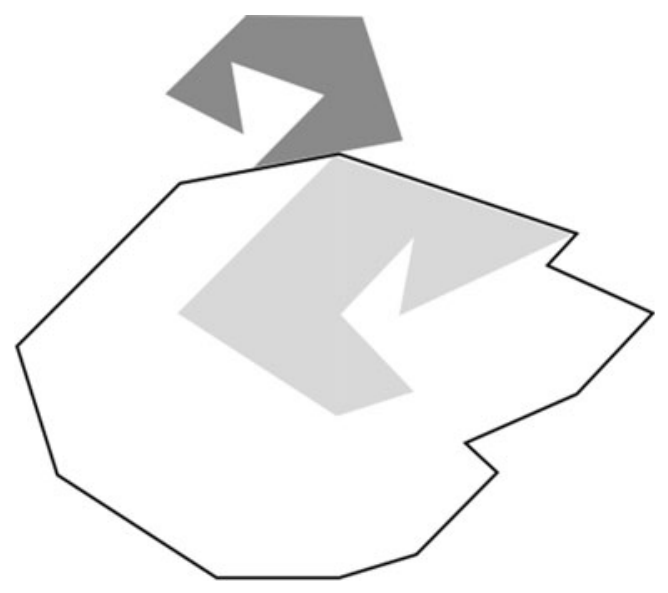

Fig. 2 No fit polygon
Dean et al. [10] presented an extension of the algorithm given by Ghosh, using a customized slope diagram. Ghosh's method works well when simple polygons with no holes are considered and when polygon $\mathrm{A}$ is nonconvex and polygon B is convex. The method also works even if both polygons are non-convex, as long as no two cavities from either polygon interferes which each other. Some difficulties using Gosh's method led Bennell et al. [11] to develop a different approach able to exploit the fact that the NFP of a non-convex polygon and a convex polygon can be easily and efficiently found by Ghosh's method. Even Bennell's method works well if the edges in a B cavity occur in the right slope order. It has to be pointed out that if the B edges within a cavity are out of right slope order an incorrect NFP is occasionally calculated [10].

\section{The no fit polygon and the no fit path}

Although NFP is a powerful geometric instrument, used and tuned by many authors in order to evaluate layout accuracy in nesting problems, when shapes are particularly complex, most of the algorithms described in literature are unable to cope with all the cases.

The following cases create a number of troubles to traditional methods when trying to solve nesting problems:

- interlocking concavities, when the orbiting polygon could be placed in a concavity of the stationary polygon although the entry is too narrow

- exact fit, when the orbiting polygon can enter inside a concavity in the stationary polygon sliding on it

- jigsaw pieces, also known as "lock-and-key" case, when the orbiting polygon ties in one hole of the stationary one

Given the complexity of obtaining the NFP when concavities between polygons interact, an alternative approach is to decompose them into a number of more manageable shapes. For this reason, the efficiency of the methods of decomposition and re-composition is very important and fundamental [14-16].

Argawal et al. [14] implemented a decomposition method based on different algorithms able to find solutions both optimal and optimized by using heuristics. They used different methods in order to decompose and obtain nonconvex polygons:

- The triangulation method searches for a pair of vertices $p_{i}$ and $p_{j}$ such that the segment $p_{i} p_{j}$ is a diagonal, namely it lies inside the polygon. It adds such a diagonal, splits the polygon into two sub-polygons by this diagonal, and triangulates each sub-polygon recur- 
sively. The procedure stops when the polygon becomes a triangle.

- Convex decomposition without Steiner points uses the same method previously presented, except that it stops as soon as the polygon does not have a reflex vertex (A vertex $\mathrm{V}$ of a polygon is a reflex vertex if its internal angle is strictly greater than $\pi$ ).

- Convex decomposition with Steiner points given a direction $e$, we extend a segment in directions $e$ and $-e$ from each reflex vertex of the polygon until it hits the polygon boundary. The result is a decomposition of the polygon into convex slabs.

Almost all the decomposition methods found in literature are quite complex, heavy and slow, and require powerful computers and sometimes the obtained NFPs have more edges than the necessary.

Finally it is possible to obtain a positive solution of the search for the NFP of non-convex polygons by using a number of appropriate tools, some of which can be found in scientific literature:

- a "customized polygons decomposition" method

- No Fit Polygon (NFP) for convex polygons

- a union-making tool for sub-polygons

- some routines able to overcome the above mentioned degenerated cases (Figs. 3, 4, 5)

Since NFP faults when some complex polygons are used (i.e. when polygons degenerate in a single line or just a point), in this work a special and complex geometric object, called "No Fit Path" (NFPh) is adopted instead of "No Fit Polygon" (NFP). The NFPh has been obtained through a specific decomposition procedure based on a tailored software previously developed by the Authors $[15,16]$.

As a matter of fact, NFPh could be defined by:

- one or more simple polygons

- two or more overlapping segments

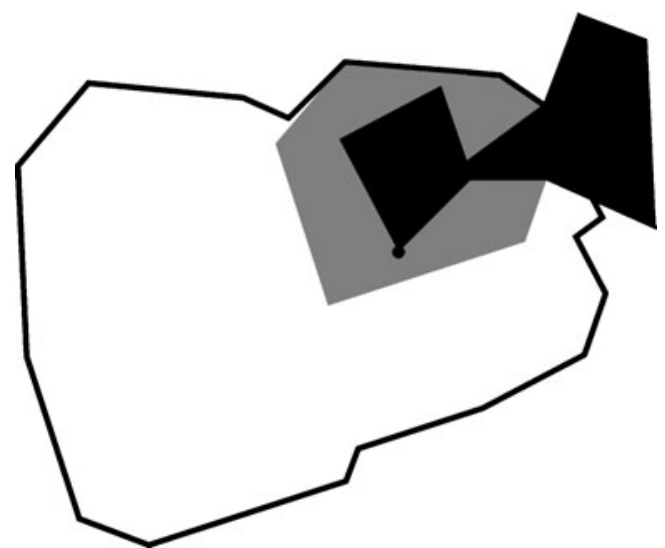

Fig. 3 Jigsaw pieces

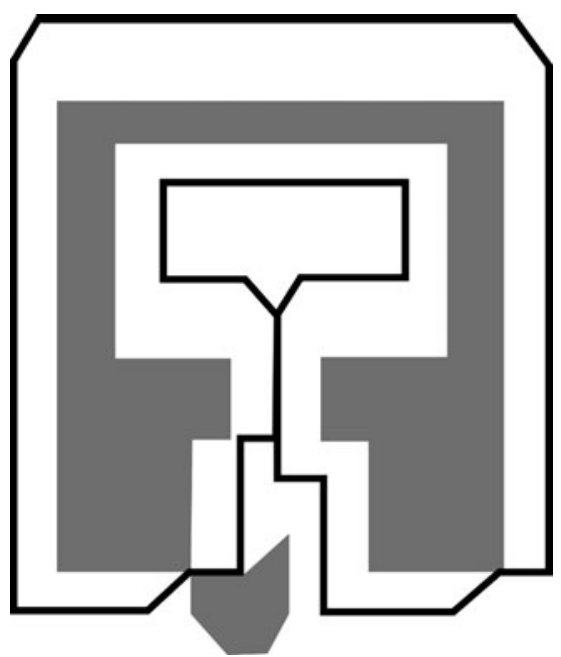

Fig. 4 Exact fit

- just some single points

- these situations all together

Given two non-convex polygons, the algorithm is able to both efficiently decompose the polygons and very quickly calculate without any approximation their NFPh by a polygon clipping method.

Using the No Fit Path concept, a data interpretation and sharing procedure has also been implemented in order to allow researchers to easily read, modify, or share their results, this way overcoming all those problems arising from the usual large amount of information and from the different origins and formats of the obtained data.

As a matter of fact, a new data format, called NestingXML [17], has been proposed in order to both overcome information fragmentation and make information sharing possible. It is a format for the representation of all data describing a nesting problem. This format is based on $\mathrm{XML}$ and was proposed to be used by researchers of the

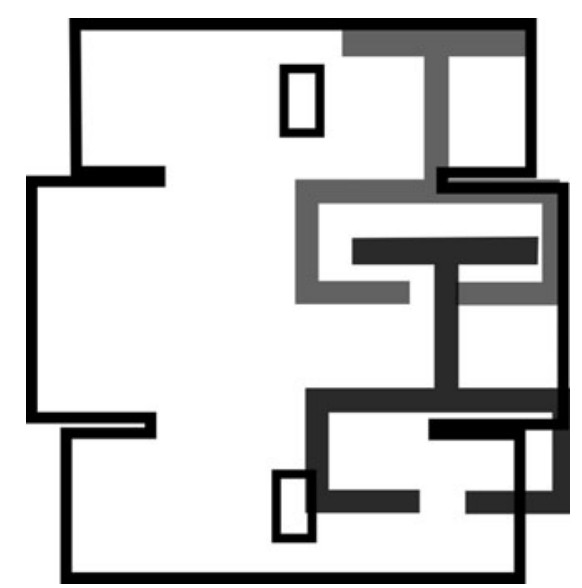

Fig. 5 Interlocking concavities 


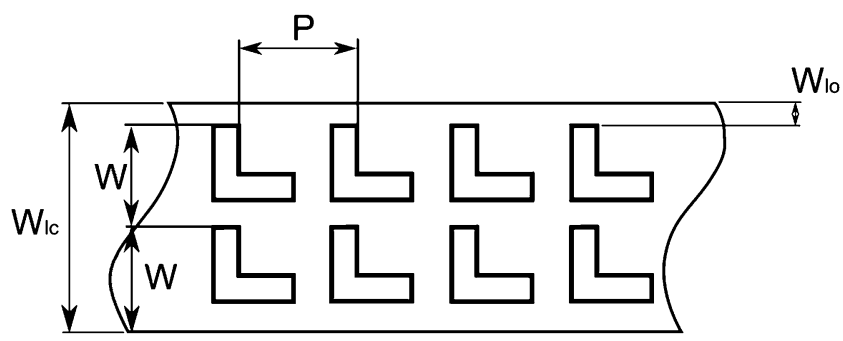

Fig. 6 Explanation of used terms

EURO Special Interest Group on Cutting and Packing for the test problem repository. The information considered in this format includes not only the original polygonal data but also the no-fit-polygons description and information about best solutions.

NestingXML is based on the XML format, which is easy to be read and understood by humans, but has a hard limit when polygons and shapes generate a No Fit Polygon with overlapped edges or when it becomes just a single point. The authors considered some changes also in the NestingXML structure, in order to add a new section where No Fit Paths can be completely stored.

In order to make an easy graphical and animated representation possible without using any commercial CAD system, an automatic routine has been developed, able to carry out a graphic output of both NFPh and orbiting and stationary polygons. Through this routine all the information about NFPh will be arranged in an XML file, easy to be managed and to be shared with other researchers.

Moreover SVG, an XML-based graphic format, very easy to be used and able to show animations and movements, has been adopted thanks to an easy-to-use interface, able to carry out an animated image, showing not only the stationary and orbiting polygon, but also their resulting NFPh. SVG is a free and open format which can be read by using just a web browser: the hardware and software platforms used do not matter in order to display these

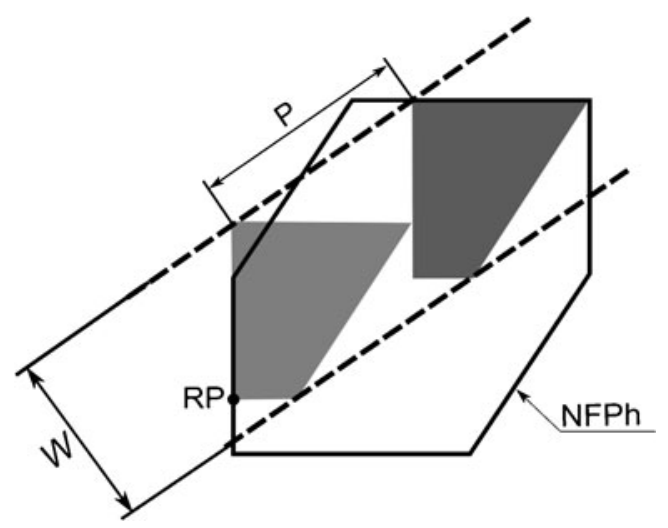

Fig. 7 Pitch and width searching
Table 1 Tests results of NFPh calculation
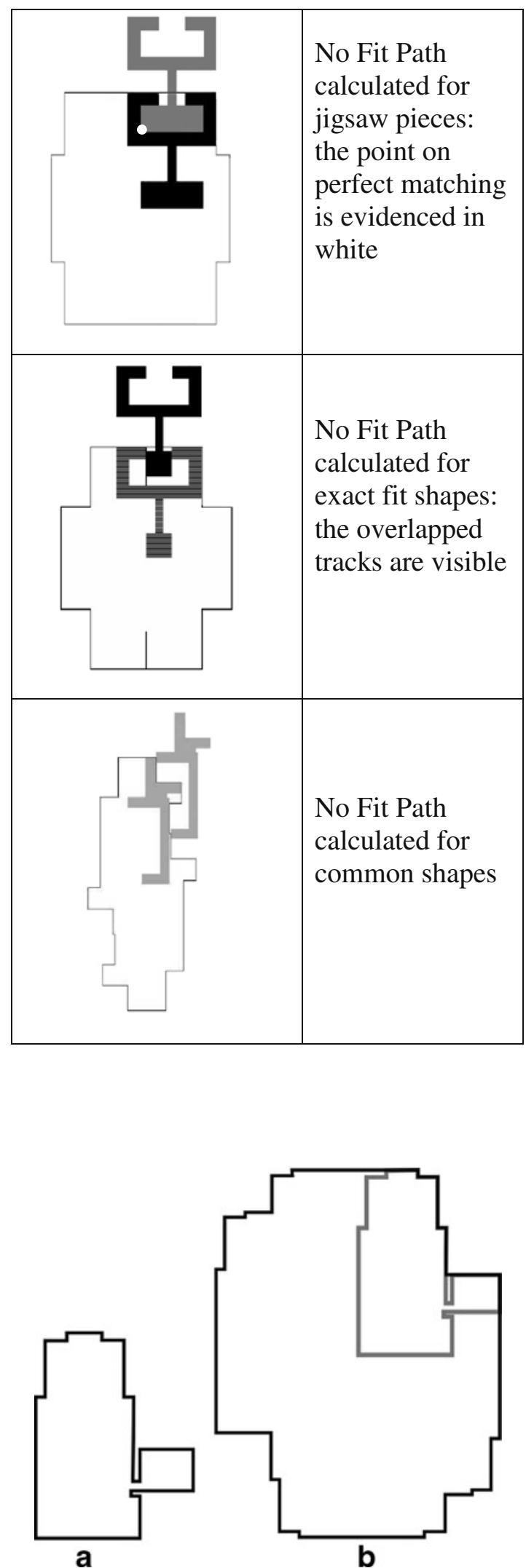

Fig. 8 The shape used in comparison of results with a Ref. [5] and b the resulting NFPh 
Table 2 Comparison of results (coil width $=1,000 \mathrm{~mm}$ )

\begin{tabular}{lccllc}
\hline & Strip pitch & Width & $u$ & $u$ & Optimal angle \\
\hline Present work & 38.47 & 57.05 & $75.2 \%$ & $72.9 \%$ & $57.1^{\circ}$ \\
Ref. [5] & 47.49 & 51.41 & $67.6 \%$ & $62.56 \%$ & $63.08^{\circ}$ \\
\hline
\end{tabular}

animations. It is a platform for two-dimensional graphics made of two parts: an XML-based file format and a programming API for graphical applications.

All the figures of NFPh in this paper have been easily obtained using the software developed by the authors without the need of any commercial application.

Given two non-convex polygons, the software developed and used in this work is able:

- to very quickly calculate without any approximation their NFPh by a polygon clipping method developed by the authors

- to carry out a clear and immediate animation of stationary polygons, orbiting polygons, and their NFPh

- to make all information about optimal positioning available

- to impose an orientation on shapes in accordance with the grain orientation

\section{Proposed method}

The shape of a blank is designed in order to achieve some targets and its total cost includes material cost, the die, the labor, and all the needed machines. It is possible to try to reduce costs when interlocking or pair-wise shapes are admissible solutions. In order to find the best configuration of strip layout, it is essential to choose the factors which are relevant to the particular situation at hand. Normally designers consider the following factors: material utilization, die costs, stamping operational cost, nature of stock (strip or roll of metal), bridge width, available space in the die set, and so on.

Considering the particular problem of cutting shapes from a roll of metal and that of stamping strip layout, there are several proposals in literature [5-7, 18-23], but they do not take into consideration the commercial strip width. In some cases the proposed methods do not properly work and

Table 3 Comparison of results (coil width $=500 \mathrm{~mm}$ )

\begin{tabular}{lcclll}
\hline & Strip pitch & Width & $u$ & $u$ & Optimal angle \\
\hline Present work & 41.72 & 55.55 & $71.2 \%$ & $71.2 \%$ & $59.9^{\circ}$ \\
Ref. [5] & 47.49 & 51.41 & $67.6 \%$ & $62.56 \%$ & $63.08^{\circ}$ \\
\hline
\end{tabular}

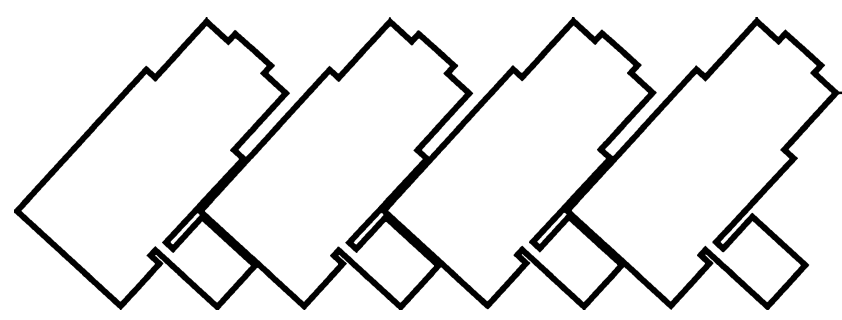

Fig. 9 The layout of the blank

the polygons' decomposition in convex sub-polygons is not well performed [7].

Unlike a number of proposed methods, in this work the commercial width of the roll of metal is considered, in order to take into account the real configuration of the problem. One of the most important steps in the real industrial environment is the optimal positioning of the shapes on a commercial roll of metal in order to cut them as economically as possible. This target has to be obtained respecting the stock left-over, the correct bridge width, the grain orientation, and a number of other important factors (Fig. 6).

As already said, the main target is to look for an optimal solution in order to minimize the waste of materials, by respecting all these restrictions; up to now a typical marker is the utilization ratio $(u)$, which has been evaluated using the following equation:

$u=\frac{A}{P W}$

where

$A \quad$ is the blank area

$P \quad$ is the pitch

$W$ is the width of a single strip

This equation is formally correct but does not take into account the commercial width of rolls of metal. A better definition of the real utilization ratio $\left(u^{\prime}\right)$ [3] could be (see also Fig. 6)

$u^{\prime}=\frac{n A}{P W_{l c}}$

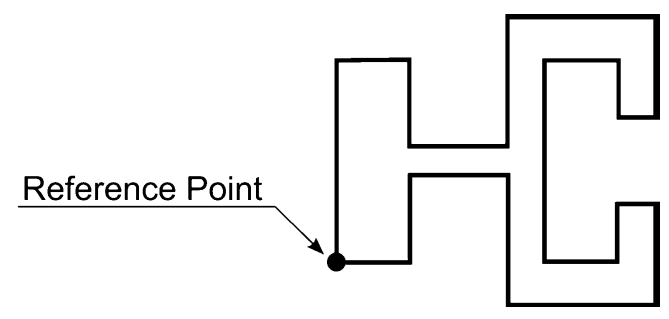

Fig. 10 The blank used in the test 


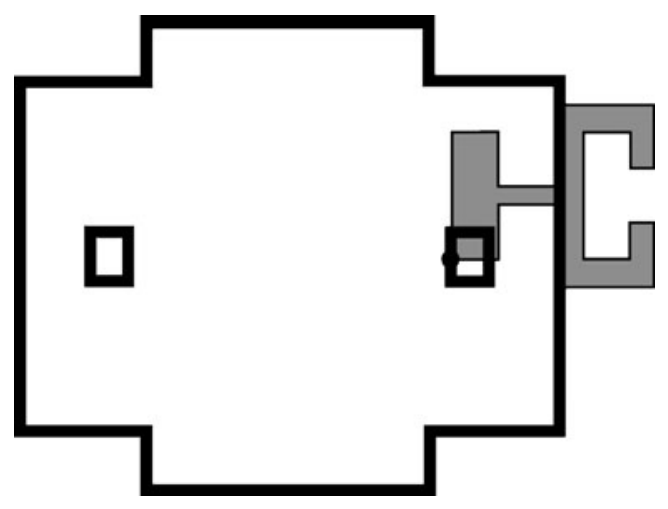

Fig. 11 The NFPh of the blank. The marked point is the optimal positioning point

where

$n=\operatorname{int}\left[\frac{W_{l c}}{W}\right]$

$W_{\text {lo }}$ is the width of left-oversand the width of the waste material strip is

$W_{\mathrm{lo}}=W_{l c}-n W$

Thanks to the method presented in the paragraph 3 , it is possible to easily obtain the NFPh of regular/irregular or convex/non-convex shapes. As a matter of fact the routines developed by the authors are able to:

- decompose non-convex shapes

- find the NFP of every pairs of convex polygons

- make union of sub-polygons

- calculate the final NFPh

The search for an optimal solution is obviously restricted to the points on the NFPh contour. As a matter of fact, the $\mathrm{NFPh}$ contour represents the location of the smallest pitch for a given position of the blank corresponding to a strip width (Fig. 7).

Once NFPh is obtained, it is possible to calculate the optimal position of the shapes on the roll of metal by using the vertex coordinates of both shapes and NFPh in order to find the optimal pitch $(P)$ between a shape and the following one.

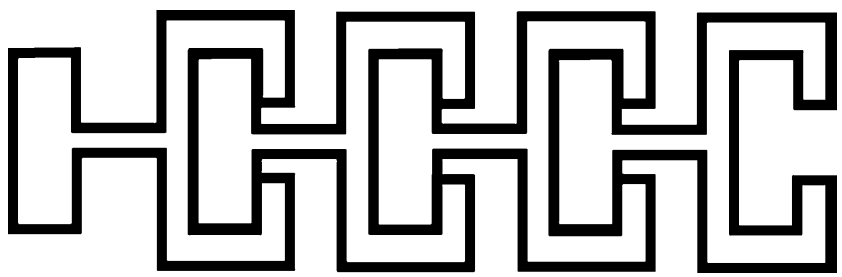

Fig. 12 The layout of the blank

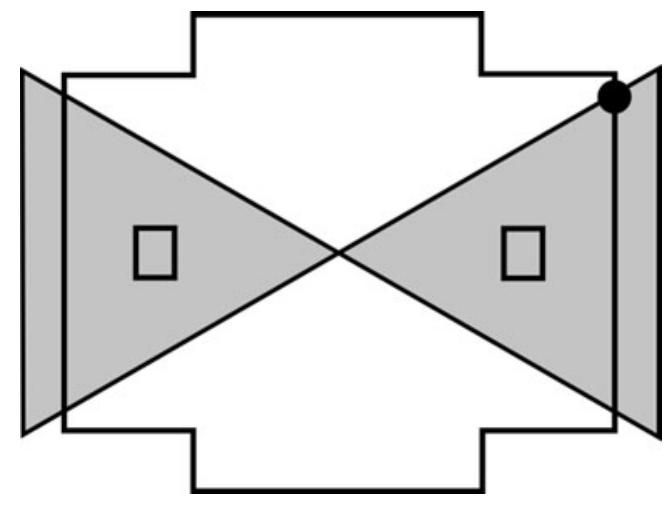

Fig. 13 The new NFPh of the blank. The marked point is the new optimal positioning point

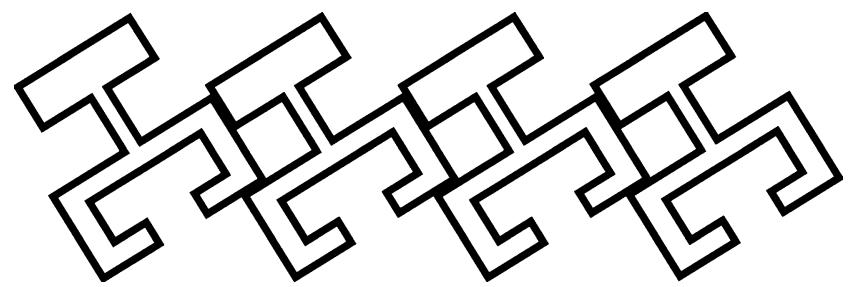

Fig. 14 The new layout of the blank

Table 4 Comparison of results changing the angle of layout

\begin{tabular}{|c|c|c|c|c|}
\hline & Angle & Pitch & Width & $\mathrm{u}^{\prime}$ \\
\hline 咆氙 & $0^{\circ}$ & 70.00 & 100.00 & 0.542 \\
\hline 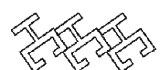 & $30^{\circ}$ & 96.04 & 136.65 & 0.314 \\
\hline
\end{tabular}

For every point of NFPh, the distance $P$ from two reference points is the searched pitch. When $P$ is known, $W$, the width of the single strip, can be obtained: it is the distance between two lines parallel to $P$ touching the upper and lower polygon vertices (see Fig. 7).

Once $P$ and $W$ are obtained, $W_{\text {lo }}$ can be calculated and all the elements are available: now the real utilization ratio can be calculated in order to maximize it.

If the shape cut from the roll of metal has to be folded in a second time, it is essential to respect the grain orientation 
of the stock, in order to assure the highest piece resistance. The proposed method makes it easy to find a solution only inside the admissible NFPh sector where the angle between the bending edge and the grain direction is lower than a fixed limit.

So, the proposed method proceeds as follows:

- NFPh computation between two identical shapes

- deletion of all the existing positions on NFPh unfitting the orientation constraints

- between all the admissible positions, the algorithm searches the optimal one, which is able to both obtain the maximum value of $u$ ' and minimize the global waste

\section{Tests and results}

In order to carry some benchmarks out, a set of tests was performed. The results of optimal disposition will follow together with corresponding items. The tests concern shapes presenting interlocking, exact fit, and jigsaw pieces. Most times the optimal solution is evident (especially in the case of interlocking), but, introducing an orientation constraint, it is possible to find different admissible positions. In the following table the results of the algorithm behavior while processing selected tests are shown (Table 1):

A number of other tests have been performed to compare results with those of other authors, like [7]. The following pictures show the considered item, the corresponding $\mathrm{NFPh}$, and a resulting strip layout (Fig. 8):

The following tables show the comparison between two methods, using different commercial strip widths (500 and 1,000 $\mathrm{mm}$, Tables 2 and 3).

It is possible to notice that the method proposed in this work not only allows designers to take the commercial strip width into account, but also to reach a higher utilization ratio (Fig. 9).

This gain could become very relevant, considering the usually large number of identical shapes produced.

Another test has been carried out in order to verify the behavior when a preferential angle $\left(30^{\circ}\right)$ is imposed (Fig. 10).

At the beginning of the test, the software calculates the NFPh of the blank without considering any angle (Fig. 11).

The software is able both to calculate the optimal disposition on the strip and to carry out a graphic output in order to display the best possible layout (Fig. 12)

The next step was to impose an angle equal to $30^{\circ}$. The algorithm calculates again the NFPh and marks the nonadmissible area, choosing a new optimal positioning point. A new solution is carried out and new graphic outputs are available (Figs. 13 and 14).
The following table finally shows and compares all the results of tests (Table 4).

It has to be highlighted how utilization ratio $u$ ' decreases when an orientation is imposed.

\section{Conclusions}

Unlike a number of proposed methods, in the evaluation of the optimal disposition of shapes on a metal strip, it has been noticed here that the best solution not always matches with the lowest utilization ratio $(u)$. As a matter of fact, the solution can be different if two technological constraints are introduced: real width of commercial roll of metal and the need for aligning the shapes on the roll of metal in accordance with the grain orientation. The proposed method uses a custom-tailored algorithm for the calculation of a complex geometric object called No Fit Path (NFPh) in order to overcome some important limits of the traditional methods, which are based on the No Fit Polygon. Tests demonstrate how more efficient this method is than the traditional ones. The utilization of this algorithm has been tested through a comparison with other procedures proposed in literature. Its results show how efficient it is in terms of optimal solution and computing time, too. It has been tested in cases of degenerate NFPh, including large numbers of edges, exact sliding, and jigsaw type fits with satisfying results.

In this paper a totally automated procedure has been used to obtain a characteristic geometric object, called "No Fit Path" (NFPh). A specific decomposition procedure has been used, based on a tailored software developed by the authors. The target was to obtain an optimal disposition of irregular shapes on a commercial roll of metal respecting the stock left-over, the correct bridge width, the grain orientation, or other important factors.

Given two non-convex polygons, this software is able to very quickly calculate without any approximation their NFPh by a number of customized routines.

After this first step, between all the existing positions on the $\mathrm{NFPh}$, the algorithm searches for the optimal one, which minimizes the global waste respecting all the imposed constraints (the stock left-over, the correct bridge width, the grain orientation, or other important factors).

This method has been compared with recent works and has proved its efficiency. The algorithm has been implemented for stamping die strip optimization and is able to consider both the commercial roll of metal width and the grain orientation in order to perform an optimal disposition of the shapes to be cut.

Acknowledgments This work was performed with the financial support of the Italian Ministry of University and Research. 


\section{References}

1. Wascher G, Haussner H, Schumann H (2007) An improved typology of cutting and packing problems. Eur J Oper Res 127 (3):1109-1130, Elsevier

2. Bennell J-A, Oliveira J-F (2008) The geometry of nesting problems: a tutorial. Eur J Oper Res 184:397-415

3. Lo Valvo E, Piacentini M, Ruisi V-F (1993) Computer aided blank layout optimum design. Manuf Syst 22:143-146

4. Lo Valvo E (1995) Nesting of irregular shapes on a strip in metal stamping blanks operations. Proc of II Convegno AITeM, 31-38

5. Nye T-J (2000) Stamping strip layout for optimal raw material utilization. J Manuf Syst 19:239-248

6. Nye T-J (2001) Optimal nesting of irregular convex blanks in strip via an exact algorithm. J Mach Tool Manuf 41:991-1002

7. Lam T-F, Sze W-S, Tan S-T (2007) Nesting of complex sheet metal parts. Comput Aid Des Appl 4:169-179

8. Crispin A-J, Clay P, Taylor G, Bayes T, Reedman D (2005) Genetic algorithm coding methods for leather nesting. Appl Intell 23-1:9-20

9. Rajender S, Sekhon G-S (1998) A low-cost modeller for two dimensional stamping layout. J Mater Process Technol 84:79-89

10. Dean H-T, Tu Y, Raffensperger J-F (2006) An improved method for calculating the no-fit polygon. Comput Oper Res 33:1521-1539

11. Bennell J-A, Song X (2008) A comprehensive and robust procedure for obtaining the nofit polygon using Minkowski sums. Comput Oper Res 35:267-281

12. Burke E-K, Kendall G (1999) Applying ant algorithms and the No Fit Polygon to the Nesting problem. Proceedings of the 12th Australian Joint Conference on Artificial Intelligence (AI'99), Sydney, Australia, December 6th-10th December 1999, Springer Lecture Notes in Artificial Intelligence 1747: 454-464

13. Watson P-D, Tobias A-M (1999) An efficient algorithm for the regular packing of polygons in the infinite plane. J Oper Res Soc 50:1054-1062

14. Agarwal P-K, Flato E, Halperin D (2000) Polygon decomposition for efficient construction of Minkowski Sums. Proc of ESA 8th Annual European Symposium on Algorithms

15. Lo Valvo E, Licari R (2007) A more efficient method for clustering sheet metal shapes. Key Eng Mater 344:921-927

16. Lo Valvo E, Licari R (2008) A proposal for a new scheme for No Fit Polygon using XML. 5th ESICUP Meeting

17. http://paginas.fe.up.pt/ esicup/tiki-index.php?page $=$ NestingXML

18. Nee A-YC (1984) A heuristic algorithm for optimum layout of metal stamping blanks. CIRP Ann 33:317-320

19. Ismail H-S, Hon KK-B (1992) New approaches for the nesting of two dimensional shapes for press tool design. Int $J$ Prod Res 30:825-837

20. Joshi S, Sudit M (1994) Procedures for solving single-pass strip layout problems. IIE Trans 26:27-37

21. Prasad YKD-V, Somasundaram S, Rao KP (1995) A sliding algorithm for optimal nesting of arbitrarily shaped sheet metal blanks. Int J Prod Res 33(6):1505-1520

22. Rajender S, Sekhon G-S (1996) A computerized diagraph and matrix approach for evaluation of metal stamping layout. J Mater Process Technol 59:285-293

23. Vamanu V, Nye T-J (2002) Stamping die strip optimization for paired parts. Proc ASME, Manuf Eng Div 13:91-96 